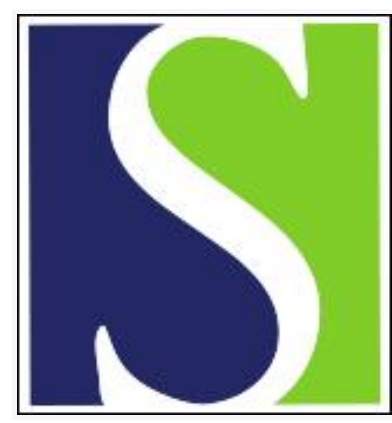

Scand J Work Environ Health 1997;23(2):130-139

https://doi.org/10.5271/sjweh.190

Issue date: Apr 1997

Associations between self-rated psychosocial work conditions and musculoskeletal symptoms and signs

by Toomingas A, Theorell T, Michélsen H, Nordemar R, Stockholm MUSIC I Study Group

The following articles refer to this text: 2001;27 suppl 1:1-102;

2008;34(6):430-437

Key terms: body regions; epidemiology; job strain; medical examination; nerve compression; psychological demand; risk estimate; social support; syndrome; tenderness

This article in PubMed: www.ncbi.nlm.nih.gov/pubmed/9167236

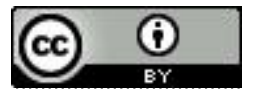




\title{
Associations between self-rated psychosocial work conditions and musculoskeletal symptoms and signs
}

\author{
by Allan Toomingas, MD, ${ }^{1,2}$ Töres Theorell, MD, ${ }^{3-5}$ Hans Michélsen, PhD, ${ }^{6}$ Rolf Nordemar, MD, ${ }^{7}$ \\ Stockholm MUSIC I Study Group ${ }^{2-11}$
}

\begin{abstract}
Toomingas A, Theorell T, Michélsen H, Nordemar R, Stockholm MUSIC I Study Group. Associations between selfrated psychosocial work conditions and musculoskeletal symptoms and signs. Scand $J$ Work Environ Health $1997: 23(2): 130-9$.

Objectives The aim was to study the associations between self-rated psychosocial work conditions and the characteristics and location of musculoskeletal symptoms, signs, and syndromes.

Methods Perceived psychosocial work conditions were recorded in a cross-sectional study with 358 men and women in various occupations. Symptoms were recorded from the musculoskeletal system with a questionnaire, and signs were detected in a medical examination of all body regions. The analyses of statistical associations between the psychosocial factors and musculoskeletal disorders were performed with control for age, gender, and physical load.

Results The most consistent and pronounced associations were mainly seen between poor psychosocial work conditions and coexisting symptoms and signs of the neck and back regions. Poor psychosocial work conditions were more consistently and strongly associated with signs of muscular (soft tissue) tenderness than with signs of tenderness in the joints, tendons, or muscular insertions or signs in nerve compression tests. Mainly low social support at work, but also high psychological demands and high job strain, were associated with such symptoms and signs, whereas decision latitude at work showed few associations with musculoskeletal disorders.

Conclusions Perceived poor psychosocial work conditions are statistically associated mainly with symptoms and signs of muscular tenderness in the central body regions. Studies on associations between psychosocial work conditions and musculoskeletal disorders should separate effect measures of different clinical signs and different body regions in order to avoid attenuation of the risk estimates.
\end{abstract}

Key terms body regions, epidemiology, job strain, medical examination, nerve compression, psychological demands, risk estimate, social support, syndromes, tenderness.

Many different risk factors have been described for the development of work-related musculoskeletal disorders in the neck and upper extremities $(1,2)$. There is a growing awareness of the importance of psychosocial work conditions (3).
The definitions and use of the term "psychosocial" diverge. One recently published definition is the following: "Psychosocial factors at work describe how the work organization is perceived by workers and managers; work organization is the objective nature of the

1 Karolinska Institute, Department of Medicine, Division of Occupational and Environmental Medicine, Huddinge, Sweden.

2 National Institute for Working Life, Department of Ergonomics, Solna, Sweden.

3 National Institute of Psychosocial Factors and Health, Solna, Sweden.

4 Karolinska Institute, Department of Clinical Neurosciences, Division of Stress Research, Solna, Sweden.

5 Karolinska Hospital, Department of Occupational Medicine, Solna, Sweden.

6 Karolinska Hospital, Department of Occupational Medicine, Solna, Sweden.

7 Karolinska Hospital, Department of Rehabilitation and Physical Medicine, Solna, Sweden.

8 Karolinska Hospital, Department of Orthopaedics, Solna, Sweden.

9 Karolinska Hospital, Department of Rheumatology, Solna, Sweden.

10 National Institute for Working Life, Department of Occupational Health, Solna, Sweden.

11 Karolinska Institute, Institute of Environmental Medicine, Solna, Sweden.

Reprint requests to: Dr Allan Toomingas, Department of Ergonomics, National Institute for Working Life, S-171 84 Solna, Sweden. 
work process and it deals with the way in which work is structured and processed [p 11]" (2). Different organizational factors are described in the literature (4). Karasek \& Theorell (5), and later Johnson \& Hall (6), have described a model for psychosocial exposure conditions including mental demands, level of control and social support at work. These variables in the "demand-control-support" model, originally found to be risk factors for cardiovascular diseases, have lately been used in the exploration of psychosocial work conditions in epidemiologic studies of work-related musculoskeletal disorders $(3,7,8)$. However, most of these studies have only recorded symptoms from selected or aggregated regions of the body, and only a few report signs from medical examinations.

The causal relations between unfavorable psychosocial work conditions and work-related musculoskeletal disorders are unclear. Pain and disorders secondary to frequent or long-lasting muscular tension or static contractions without pause, as a reaction to mentally stressful exposure and not due to a physical load, constitute a common theory $(7,9-12)$. The theory is however not yet developed enough to specify where the tension and pain would be localized in the body. In 1933 one of the pioneers of psychosomatics, Wilhelm Reich, described a "muscular armor" with a segmental distribution of tensed muscles in the face, neck, trunk, and pelvis as a reaction to deep frustration (13). Symptoms among workers with psychosocial problems are reported to be restricted mainly to the neck and shoulder regions (14). Elevated electromyographic (EMG) activity has been recorded from the neck, trapezius, and erector spine muscles during stress provocation among patients with pain syndromes in the cervical or back regions (15-19). Elevated EMG activity has also been reported from the trapezius muscles during experimentally induced mentally demanding tasks $(20-24)$. The theory of stress-induced muscular tension or the lack of ability to relax as a mediator of the effects of poor psychosocial work conditions predicts the findings of muscular pain and tenderness among subjects working in such conditions. Symptoms and signs from the muscular insertions, tendons, or joints or those indicating nerve entrapment are not primarily consistent with this theory, as affections in these structures are thought to be associated mainly with high, long-lasting, or repetitive physical load (2, pp 24-80).

More knowledge of the characteristics and distribution of the symptoms and signs associated with poor psychosocial work conditions would, it is hoped, contribute to a better understanding of these disorders.

The objective of this study was to examine the associations between self-rated psychosocial work conditions and the characteristics and location of musculoskeletal symptoms, signs, and syndromes.

The following hypotheses were formulated: (i) the symptoms, signs, and syndromes associated with high psychosocial work exposure are located in central body regions (neck, scapula or back) rather than in peripheral regions (arms, hands, legs or feet), (ii) the findings of medical examinations in association with high psychosocial work exposure are signs of muscular (soft-tissue) tenderness rather than signs of affections of nerves, joints, muscular insertions, and tendons.

\section{Subjects and methods}

This study was part of a methodological project, the major objective of which was to develop and evaluate different methods of measuring exposure and effect in epidemiologic studies of work-related musculoskeletal disorders (25). The study group of 358 subjects (participation rate $71 \%$ ) included 83 male furniture movers (median age 35 years), 89 female medical secretaries (41 years), and 96 men (41 years) and 90 women (43 years) of the working population. The study was approved by the regional ethical research committee.

\section{Psychosocial variables}

The subjects rated the psychosocial work conditions of their present work according to 17 questions in a questionnaire with 4-point scales $(5,26) .{ }^{I 2}$ Ratings were transformed into indices representing the psychosocial exposure variables "psychological demands" (5 questions), "decision latitude" (6 questions), and "social support" (6 questions). "Job strain" was defined as the ratio between "psychological demands" and "decision latitude" (27). Subjects were categorized for each of the 4 psychosocial indices into a "low", "medium", or "high" exposure group by cut-off points approximately at the 30 33rd and 67-70th percentiles, respectively ("social support" at the 20 th and 73 rd due to the discrete scale).

\section{Symptoms}

Symptoms (at present and during the past 12 months) of the neck, shoulders, elbows or forearms, wrists or hands, thoracic back, lumbar back, hips, and knees, ankles or feet were recorded by means of a slightly modified version of the Nordic questionnaire (28). ${ }^{13}$

\section{Signs}

The subjects were assigned to the medical examination in a randomized fashion by 1 of a team of 8 physicians blinded to all information about the subject's exposure 
Table 1. Psychosocial exposure variables for the whole study group and for the subgroups used for stratification: gender, age group and physical

\begin{tabular}{|c|c|c|c|c|c|c|c|c|c|c|c|c|c|c|c|}
\hline \multirow{3}{*}{$\begin{array}{l}\text { Psychosocial } \\
\text { exposure } \\
\text { variable }\end{array}$} & \multirow{2}{*}{\multicolumn{3}{|c|}{ All subjects }} & \multirow{2}{*}{\multicolumn{3}{|c|}{ Women }} & \multirow{2}{*}{\multicolumn{3}{|c|}{ Men }} & \multicolumn{6}{|c|}{ Age-group } \\
\hline & & & & & & & & & & \multicolumn{3}{|c|}{$20-44$ years } & \multicolumn{3}{|c|}{$45-64$ years } \\
\hline & $N$ & Mean & SD & $N$ & Mean & $\mathrm{SD}$ & $\mathrm{N}$ & Mean & SD & $\mathrm{N}$ & Mean & $S D$ & $N$ & Mean & SD \\
\hline $\begin{array}{l}\text { Psychological } \\
\text { demands } \\
\left(\mathrm{R}_{\max }=5-20\right)\end{array}$ & 339 & 12.1 & 2.68 & 169 & 12.3 & 2.71 & 170 & 11.9 & 2.64 & 212 & 12.1 & 2.58 & 127 & 12.1 & 2.84 \\
\hline $\begin{array}{l}\text { Decision } \\
\text { latitude } \\
\left(\mathrm{R}_{\max }=6-24\right)\end{array}$ & 339 & 17.8 & 3.11 & 170 & 17.1 & 3.04 & 169 & 18.6 & 3.00 & 211 & 17.6 & 3.07 & 128 & 18.2 & 3.15 \\
\hline $\begin{array}{l}\text { Social } \\
\text { supportb } \\
\left(R_{\max }=1-4\right)\end{array}$ & 329 & 3.26 & 0.493 & 162 & 3.26 & 0.512 & 167 & 3.27 & 0.476 & 207 & 3.23 & 0.468 & 122 & 3.32 & 0.531 \\
\hline $\begin{array}{l}\text { Job strain }^{\mathrm{a}} \\
\left(\mathrm{R}_{\max }=0.21-3.33\right)\end{array}$ & 338 & 0.704 & 0.224 & 169 & 0.750 & 0.245 & 169 & 0.659 & 0.192 & 211 & 0.716 & 0.227 & 127 & 0.684 & 0.218 \\
\hline
\end{tabular}

a Exposed $=$ high values

Exposed $=$ low values.

situation or symptoms. It followed a strict protocol and covered the musculoskeletal system of the entire body. The team had received special training in examination methods and evaluation during practical seminars with an experienced orthopedic surgeon as a tutor, and they had used a video-recorded examination as a "standard" in order to enhance reliability and validity. Interexaminer reliability was studied in a randomized subgroup (47 subjects). It showed kappa coefficients from 0.52 0.62 for tests of tenderness or pain on resisted muscular contraction, range of joint motion, or nerve entrapment. Twenty-four items were selected for this study, covering all body regions, as were the following examination moments: inspection; palpation of muscular (soft-tissue) tenderness; palpation of tenderness in the tendons, muscular insertions or joints; tests of joint movement restriction; tests of pain on resisted muscular contraction; tests of nerve function - signs of nerve compression or decreased sensitivity. "Spine muscle tenderness" was constructed as an item summarizing the signs of tenderness in the extensor muscles of the neck, back or descending trapezius. "Joint tenderness" summarized signs of tenderness in muscular insertions, tendons, or joints in the upper and lower extremities or the back. "Nerve affections" summarized positive signs in the nerve compression tests of the cervical spine or upper extremities and decreased sensitivity in the lower extremities. A few extra items less likely to be associated with occupational exposure were also included as a check of the validity of the results (shoulder asymmetry, wrist-joint laxity, hip rotation range and hallux valgus).

\section{Syndromes}

Data on present symptoms and signs were combined into the following 5 syndromes: "tension neck", "nerve com- pression syndrome", "tendalgia of the upper extremities", "lumbalgia" and "tendalgia of the lower extremities". Is

\section{Statistical methods}

In order to control for variations in occupational physical load, data analyses were done stratifying for questionnaire-based data on physical load on the musculoskeletal system in the neck, upper extremities, low back, and lower extremities. The prevalence ratios (PR) with testbased $95 \%$ confidence-intervals $(95 \% \mathrm{CI})$ for data stratified by age ( 2 groups: $20-44$ and $45-64$ years), gender, and physical load (low and high) according to Mantel-Haenszel were calculated with symptoms, signs, or syndromes as effect variables $(29,30)$. In order to analyze existing linear dose-effect relations, the prevalence ratios were calculated comparing the high and low exposure groups ( $\mathrm{PR}_{\text {high/low }}$ ) and the medium and low exposure groups $\left(\mathrm{PR}_{\text {mediumllow }}\right)$. Analyses were done using the SAS computer program (proc freq) (SAS Institute, North Carolina, United States).

In order to emphasize the pronounced associations between the psychosocial exposure variables and the effect variables, the following crude criteria were established: prevalence of effect variable at least $10 \%$ (not applied to "syndromes" due to the low prevalences); $\mathrm{PR}_{\text {higl/low }}$ at least 1.5 with $95 \%$ confidence interval above $1.0 ; \mathrm{PR}_{\text {medium/low }}$ above $1.0 ; \mathrm{PR}_{\text {high/low }}$ higher or equal to $\mathrm{PR}_{\text {medium/low. }}$

\section{Results}

The frequency distributions of the psychosocial indices were fairly normal. The variation in psychosocial expo-

14 Description of the items and the specification of the criteria for positive signs obtainable from the senior author.

15 Specification of the criteria obtainable from the senior author. 
load. $\left(R_{\max }=\right.$ maximum possible range $)$

\begin{tabular}{|c|c|c|c|c|c|c|c|c|c|c|c|c|c|c|}
\hline \multicolumn{6}{|c|}{ Physical load } & \multicolumn{9}{|c|}{ Psychosocial exposure group } \\
\hline \multicolumn{3}{|c|}{ High } & \multicolumn{3}{|c|}{ Low } & \multicolumn{3}{|c|}{ High } & \multicolumn{3}{|c|}{ Medium } & \multicolumn{3}{|c|}{ Low } \\
\hline N & Mean & SD & N & Mean & SD & $\mathrm{N}$ & Mean & Range & $\mathrm{N}$ & Mean & Range & $N$ & Mean & Range \\
\hline 170 & 12.3 & 2.47 & 152 & 12.0 & 2.77 & 106 & 15.2 & $14-19$ & 140 & 12,0 & $11-13$ & 93 & 8.83 & $5-10$ \\
\hline 170 & 17.6 & 2.72 & 152 & 18.1 & 3.40 & 109 & 14.3 & $7-16$ & 122 & 18.0 & $17-19$ & 108 & 21.2 & $20-24$ \\
\hline 164 & 3.24 & 0.517 & 149 & 3.28 & 0.475 & 67 & 2.59 & $1.17-2.83$ & 172 & 3.20 & $3.0-3.5$ & 90 & 3.87 & $3.6-4.0$ \\
\hline 170 & 0.711 & 0.196 & 151 & 0.690 & 0.228 & 111 & 0.941 & $0.77-1.89$ & 113 & 0.679 & $0.61-0.76$ & 114 & 0.499 & $0.21-0.60$ \\
\hline
\end{tabular}

sure was high when judged from the standard deviations of the total group and the means and ranges of the high-, medium-, and low-exposure groups. Women, the lowage group, and those with high physical load tended to report somewhat more unfavorable psychosocial work conditions (table 1).

Most of the prevalence ratios were above 1.0 (ie, there were mainly positive statistical associations between unfavorable psychosocial work conditions and symptoms, signs or syndromes). "Association" in the text below is therefore used for "positive statistical association". The index "decision latitude" had very few associations with the effect variables.

The most common symptoms were reported for the neck, shoulder, and low-back regions (table 2). Pronounced associations with the psychosocial variables were seen for the neck, back, and hip regions (table 2 and figure 1). Strong associations were seen between social support and symptoms from most of the body regions except the shoulders, which showed no substantial association with any of the exposure variables.

The most frequent and pronounced signs found in the medical examination were noted from the neck, shoulder, arms, and back (table 3 and figure 1). All pronounced associations were to signs of tenderness, mainly in muscles (soft tissues). Scattered associations, however mostly not fulfilling the criteria of pronounced associations, were also seen with tenderness at muscular insertions or tendons in the rotator cuff, the lateral humeral epicondyles and the feet, with restrictions of joint movements in the neck and glenohumeral joints and with signs of nerve affection in the upper extremities and the back (decreased sensitivity in the legs). Among the summary variables, only "spine muscle tenderness" showed pronounced associations, mainly with psychological demands and job strain. No substantial associations were noted with the variables "shoulder asymmetry", "wrist joint laxity", "hip rotation range" or "hallux valgus" (data not shown here).

Associations to coexisting symptoms and signs were only found for the neck and low back, as described by the syndromes "tension neck" and "lumbalgia" (table 4). The prevalences for questionnaire-based data on present symptoms from the different regions included in the diagnostic criteria were about $35-50 \%$ of the corresponding values during the past year.

\section{Discussion}

The main results support the hypotheses of this study. The most pronounced and consistent associations were found between high mental demands or low social support and coexisting symptoms and signs of muscular (soft-tissue) tenderness in the central body regions (neck and low back), most clearly described by the "tension neck" and "lumbalgia" syndromes. Our study confirmed the findings from earlier studies regarding associations with symptoms from the neck or back regions $(12,31-$ 36). Theorell and his co-workers also found associations between demand-control-support variables and symptoms of the back, neck, or shoulder regions but not from symptoms of other joints (7). These results all disagree with the conclusions from other studies indicating " $\mathrm{a}$ general musculoskeletal sensitivity to mental stress" ( 32 , $36)$. The results from the shoulder regions are contradictory. The consistent lack of associations with symptoms from the shoulders is noteworthy and is in contrast with the results from the neighboring neck region and also with findings in another study using the same questionnaire (34). The results are also contradicted by the findings of pronounced associations with signs of tenderness and with contraction pain at the rotator cuff. Reverse but still contradictory findings were noted regarding the hip 
Table 2. Prevalences of questionnaire-based data on symptoms during the past year for 358 subjects of mixed gender and occupations. Prevalence ratios (PR) with $95 \%$ test-based confidence intervals $(95 \% \mathrm{Cl}$ ) for subjects with high exposure versus subjects with low exposure (high/low) and subjects with medium exposure versus subjects with low exposure (medium/low) to 4 variables of self-rated psychosocial work conditions. PR based on Mantel-Haenszel calculations of data stratified by gender, 2 age groups and low/high physical load at work.

\begin{tabular}{|c|c|c|c|c|c|c|c|c|c|}
\hline \multirow[t]{3}{*}{ Body region } & \multirow{3}{*}{$\begin{array}{c}\text { Preva- } \\
\text { lence } \\
(\%)\end{array}$} & \multicolumn{8}{|c|}{ Psychosocial exposure variable } \\
\hline & & \multicolumn{2}{|c|}{$\begin{array}{l}\text { Psychological } \\
\text { demands }\end{array}$} & \multicolumn{2}{|c|}{$\begin{array}{l}\text { Decision } \\
\text { latitude }\end{array}$} & \multicolumn{2}{|c|}{$\begin{array}{l}\text { Social } \\
\text { support }\end{array}$} & \multicolumn{2}{|c|}{$\begin{array}{l}\text { Job } \\
\text { strain }\end{array}$} \\
\hline & & PR & $95 \% \mathrm{Cl}$ & PR & $95 \% \mathrm{Cl}$ & PR & $95 \% \mathrm{Cl}$ & $P R$ & $95 \% \mathrm{Cl}$ \\
\hline 1 Neck & 45 & & & & & & & & \\
\hline $\begin{array}{l}\text { High/low } \\
\text { Medium/low }\end{array}$ & & $\begin{array}{l}1.5^{\mathrm{a}} \\
1.5\end{array}$ & $\begin{array}{l}1.1-2.2 \\
1.0-2.1\end{array}$ & $\begin{array}{l}1.2 \\
1.0\end{array}$ & $\begin{array}{l}0.87-1.8 \\
0.73-1.5\end{array}$ & $\begin{array}{l}1.6^{\mathrm{a}} \\
1.2\end{array}$ & $\begin{array}{r}1.1-2.3 \\
0.84-1.6\end{array}$ & $\begin{array}{l}1.6^{\mathrm{a}} \\
1.4\end{array}$ & $\begin{array}{r}1.1-2.2 \\
0.96-2.0\end{array}$ \\
\hline 2 Shoulders & 53 & & & & & & & & \\
\hline $\begin{array}{l}\text { High/low } \\
\text { Medium/low }\end{array}$ & & $\begin{array}{l}1.1 \\
1.1\end{array}$ & $\begin{array}{l}0.85-1.5 \\
0.84-1.5\end{array}$ & $\begin{array}{l}1.1 \\
1.0\end{array}$ & $\begin{array}{l}0.85-1.5 \\
0.79-1.4\end{array}$ & $\begin{array}{l}1.2 \\
1.2\end{array}$ & $\begin{array}{l}0.87-1.7 \\
0.95-1.6\end{array}$ & $\begin{array}{l}1.1 \\
1.1\end{array}$ & $\begin{array}{l}0.87-1.5 \\
0.80-1.4\end{array}$ \\
\hline 3 Elbows & 15 & & & & & & & & \\
\hline $\begin{array}{l}\text { High/low } \\
\text { Medium/low }\end{array}$ & & $\begin{array}{l}1.0 \\
1.3\end{array}$ & $\begin{array}{l}0.49-2.2 \\
0.61-2.7\end{array}$ & $\begin{array}{l}1.0 \\
0.59\end{array}$ & $\begin{array}{l}0.50-2.0 \\
0.27-1.3\end{array}$ & $\begin{array}{l}1.7 \\
0.96\end{array}$ & $\begin{array}{l}0.83-3.5 \\
0.47-2.0\end{array}$ & $\begin{array}{l}1.3 \\
1.1\end{array}$ & $\begin{array}{l}0.60-2.9 \\
0.46-2.5\end{array}$ \\
\hline 4 Wrists/hands & 26 & & & & & & & & \\
\hline $\begin{array}{l}\text { High/low } \\
\text { Medium/low }\end{array}$ & & $\begin{array}{l}1.2 \\
1.1\end{array}$ & $\begin{array}{l}0.70-2.1 \\
0.61-1.9\end{array}$ & $\begin{array}{l}1.0 \\
0.83\end{array}$ & $\begin{array}{l}0.62-1.7 \\
0.51-1.4\end{array}$ & $\begin{array}{l}1.8 \\
0.99\end{array}$ & $\begin{array}{r}1.1-3.1 \\
0.57-1.7\end{array}$ & $\begin{array}{l}1.4 \\
1.4\end{array}$ & $\begin{array}{l}0.81-2.6 \\
0.79-2.3\end{array}$ \\
\hline 5 Thoracic back & 22 & & & & & & & & \\
\hline $\begin{array}{l}\text { High/low } \\
\text { Medium/low }\end{array}$ & & $\begin{array}{l}1.2 \\
0.57\end{array}$ & $\begin{array}{l}0.72-2.1 \\
0.30-1.1\end{array}$ & $\begin{array}{l}1.2 \\
0.84\end{array}$ & $\begin{array}{l}0.72-2.2 \\
0.46-1.5\end{array}$ & $\begin{array}{l}1.8 \\
1.5\end{array}$ & $\begin{array}{l}0.94-3.5 \\
0.82-2.7\end{array}$ & $\begin{array}{l}0.96 \\
0.91\end{array}$ & $\begin{array}{l}0.56-1.7 \\
0.50-1.7\end{array}$ \\
\hline 6 Lumbar back & 57 & & & & & & & & \\
\hline $\begin{array}{l}\text { High/low } \\
\text { Medium/low }\end{array}$ & & $\begin{array}{l}1.3 \\
1.1\end{array}$ & $\begin{array}{r}1.0-1.7 \\
0.79-1.4\end{array}$ & $\begin{array}{l}1.1 \\
1.1\end{array}$ & $\begin{array}{l}0.88-1.5 \\
0.82-1.4\end{array}$ & $\begin{array}{l}1.6^{\mathrm{a}} \\
1.5\end{array}$ & $\begin{array}{l}1.2-2.2 \\
1.2-2.0\end{array}$ & $\begin{array}{l}1.2 \\
1.1\end{array}$ & $\begin{array}{l}0.94-1.6 \\
0.83-1.4\end{array}$ \\
\hline 7 Hips & 22 & & & & & & & & \\
\hline $\begin{array}{l}\text { High/low } \\
\text { Medium/low }\end{array}$ & & $\begin{array}{l}2.7^{\mathrm{a}} \\
2.1\end{array}$ & $\begin{array}{l}1.3-5.5 \\
1.1-4.2\end{array}$ & $\begin{array}{l}0.97 \\
0.85\end{array}$ & $\begin{array}{l}0.58-1.6 \\
0.51-1.4\end{array}$ & $\begin{array}{l}2.0 \\
2.0\end{array}$ & $\begin{array}{r}0.99-4.0 \\
1.1-3.7\end{array}$ & $\begin{array}{l}1.4 \\
0.96\end{array}$ & $\begin{array}{l}0.77-2.5 \\
0.52-1.8\end{array}$ \\
\hline 8 Knees & 37 & & & & & & & & \\
\hline $\begin{array}{l}\text { High/low } \\
\text { Medium/low }\end{array}$ & & $\begin{array}{l}0.97 \\
0.78\end{array}$ & $\begin{array}{l}0.66-1.4 \\
0.53-1.1\end{array}$ & $\begin{array}{l}1.1 \\
1.2\end{array}$ & $\begin{array}{l}0.72-1.6 \\
0.85-1.7\end{array}$ & $\begin{array}{l}1.5 \\
1.1\end{array}$ & $\begin{array}{l}0.99-2.4 \\
0.76-1.7\end{array}$ & $\begin{array}{l}0.82 \\
0.93\end{array}$ & $\begin{array}{l}0.56-1.2 \\
0.65-1.3\end{array}$ \\
\hline 9 rnkles/feet & 24 & & & & & & & & \\
\hline $\begin{array}{l}\text { High/low } \\
\text { Medium/low }\end{array}$ & & $\begin{array}{l}1.1 \\
0.57\end{array}$ & $\begin{array}{l}0.68-1.8 \\
0.32-1.0\end{array}$ & $\begin{array}{l}0.90 \\
1.6\end{array}$ & $\begin{array}{r}0.45-1.7 \\
1.0-2.6\end{array}$ & $\begin{array}{l}1.8 \\
1.3\end{array}$ & $\begin{array}{l}0.99-3.4 \\
0.72-2.2\end{array}$ & $\begin{array}{l}1.3 \\
0.98\end{array}$ & $\begin{array}{l}0.78-2.3 \\
0.55-1.8\end{array}$ \\
\hline
\end{tabular}

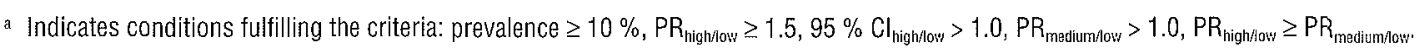

Table 3. Prevalences of positive signs in the medical examination of 358 subjects of mixed gender and occupations. Prevalence ratios (PR) with 95\% test-based confidence intervals $(95 \% \mathrm{Cl}$ ) for subjects with high exposure versus subjects with low exposure (high/low) and subjects with medium exposure versus subjects with low exposure (medium/low) to 4 variables of self-rated psychosocial work conditions. PR based on Mantel-Haenszel calculations on data stratified by gender, 2 age groups and low/high physical load at work.

\begin{tabular}{|c|c|c|c|c|c|c|c|c|c|}
\hline \multirow[t]{3}{*}{ Examination item } & \multirow{3}{*}{$\begin{array}{l}\text { Preva- } \\
\text { lence } \\
(\%)\end{array}$} & \multicolumn{8}{|c|}{ Psychosocial exposure variable } \\
\hline & & \multicolumn{2}{|c|}{$\begin{array}{l}\text { Psychological } \\
\text { demands }\end{array}$} & \multicolumn{2}{|c|}{$\begin{array}{l}\text { Decision } \\
\text { latitude }\end{array}$} & \multicolumn{2}{|c|}{$\begin{array}{l}\text { Social } \\
\text { support }\end{array}$} & \multicolumn{2}{|r|}{$\begin{array}{c}\text { Job } \\
\text { strain }\end{array}$} \\
\hline & & $P R$ & $95 \% \mathrm{Cl}$ & PR & $95 \% \mathrm{Cl}$ & $P R$ & $95 \% \mathrm{Cl}$ & $P R$ & $95 \% \mathrm{Cl}$ \\
\hline 1 Neck tenderness & 21 & & & & & & & & \\
\hline $\begin{array}{l}\text { High/low } \\
\text { Medium/low }\end{array}$ & & $\begin{array}{l}2.0^{\mathrm{a}} \\
1.1\end{array}$ & $\begin{array}{r}1.1-3.7 \\
0.60-2.2\end{array}$ & $\begin{array}{l}1.3 \\
1.1\end{array}$ & $\begin{array}{l}0.74-2.3 \\
0.64-2.0\end{array}$ & $\begin{array}{l}1.8 \\
1.6\end{array}$ & $\begin{array}{l}0.90-3.5 \\
0.89-2.9\end{array}$ & $\begin{array}{l}2.1^{\mathrm{a}} \\
1.6\end{array}$ & $\begin{array}{r}1.2-3.7 \\
0.84-3.0\end{array}$ \\
\hline 2 Neck movement restriction & 3.6 & & & & & & & & \\
\hline $\begin{array}{l}\text { High/low } \\
\text { Medium/low }\end{array}$ & & $\begin{array}{l}.6 \\
. .6\end{array}$ & $\begin{array}{l}. . \\
. .\end{array}$ & $\begin{array}{l}1.6 \\
2.3\end{array}$ & $\begin{array}{l}0.17-15 \\
0.33-16\end{array}$ & $\begin{array}{l}. b \\
. . b\end{array}$ & $\begin{array}{l}. . \\
. .\end{array}$ & $\begin{array}{l}8.3 \\
2.4\end{array}$ & $\begin{array}{l}0.93-75 \\
0.22-27\end{array}$ \\
\hline 3 Trapezius tenderness & 20 & & & & & & & & \\
\hline $\begin{array}{l}\text { High/low } \\
\text { Medium/low }\end{array}$ & & $\begin{array}{l}1.6 \\
1.2\end{array}$ & $\begin{array}{l}0.87-3.0 \\
0.66-2.3\end{array}$ & $\begin{array}{l}1.3 \\
1.2\end{array}$ & $\begin{array}{l}0.71-2.4 \\
0.65-2.1\end{array}$ & $\begin{array}{l}1.5 \\
1.4\end{array}$ & $\begin{array}{l}0.79-3.0 \\
0.79-2.4\end{array}$ & $\begin{array}{l}2.0^{\mathrm{a}} \\
2.0\end{array}$ & $\begin{array}{l}1.1-3.7 \\
1.1-3.8\end{array}$ \\
\hline 4 Rotator cuff tenderness & 12 & & & & & & & & \\
\hline $\begin{array}{l}\text { High/low } \\
\text { Medium/low }\end{array}$ & & $\begin{array}{l}2.0 \\
1.5\end{array}$ & $\begin{array}{l}0.83-4.9 \\
0.63-3.7\end{array}$ & $\begin{array}{l}1.9 \\
2.2\end{array}$ & $\begin{array}{l}0.78-4.7 \\
0.91-5.3\end{array}$ & $\begin{array}{l}3.2^{\mathrm{a}} \\
2.2\end{array}$ & $\begin{array}{r}1.3-7.8 \\
0.97-5.2\end{array}$ & $\begin{array}{l}2.2 \\
0.97\end{array}$ & $\begin{array}{l}0.96-5.1 \\
0.36-2.6\end{array}$ \\
\hline
\end{tabular}


Table 3. Continued

\begin{tabular}{|c|c|c|c|c|c|c|c|c|c|}
\hline \multirow[t]{3}{*}{ Examination item } & \multirow{3}{*}{$\begin{array}{l}\text { Preva- } \\
\text { lence } \\
(\%)\end{array}$} & \multicolumn{8}{|c|}{ Psychosocial exposure variable } \\
\hline & & \multicolumn{2}{|c|}{$\begin{array}{l}\text { Psychological } \\
\text { demands }\end{array}$} & \multicolumn{2}{|c|}{$\begin{array}{l}\text { Decision } \\
\text { latitude }\end{array}$} & \multicolumn{2}{|c|}{$\begin{array}{l}\text { Social } \\
\text { support }\end{array}$} & \multicolumn{2}{|r|}{$\begin{array}{l}\text { Job } \\
\text { strain }\end{array}$} \\
\hline & & PR & $95 \% \mathrm{Cl}$ & PR & $95 \% \mathrm{Cl}$ & PR & $95 \% \mathrm{Cl}$ & PR & $95 \% \mathrm{Cl}$ \\
\hline 5 Shoulder movement restriction & 5.9 & & & & & & & & \\
\hline $\begin{array}{l}\text { High/low } \\
\text { Medium/low }\end{array}$ & & $\begin{array}{l}3.0 \\
2.4\end{array}$ & $\begin{array}{l}0.67-14 \\
0.48-12\end{array}$ & $\begin{array}{l}0.91 \\
1.0\end{array}$ & $\begin{array}{l}0.20-4.1 \\
0.31-3.2\end{array}$ & $\begin{array}{l}1.0 \\
1.5\end{array}$ & $\begin{array}{l}0.21-4.9 \\
0.43-5.2\end{array}$ & $\begin{array}{l}1.9 \\
4.4\end{array}$ & $\begin{array}{c}0.43-8.1 \\
1.1-18\end{array}$ \\
\hline 6 Shoulder contraction pain & 6.4 & & & & & & & & \\
\hline $\begin{array}{l}\text { High/low } \\
\text { Medium/low }\end{array}$ & & $\begin{array}{l}3.0 \\
2.1\end{array}$ & $\begin{array}{l}0.82-11 \\
0.63-7.0\end{array}$ & $\begin{array}{l}0.70 \\
2.1\end{array}$ & $\begin{array}{l}0.16-3.1 \\
0.81-5.2\end{array}$ & $\begin{array}{l}6.2 \\
2.3\end{array}$ & $\begin{array}{r}1.5-25 \\
0.48-11\end{array}$ & $\begin{array}{l}4.1 \\
1.7\end{array}$ & $\begin{array}{c}1.2-14 \\
0.49-5.6\end{array}$ \\
\hline 7 Humeral epicondyl tenderness & 12 & & & & & & & & \\
\hline $\begin{array}{l}\text { High/low } \\
\text { Medium/low }\end{array}$ & & $\begin{array}{l}1.2 \\
1.0\end{array}$ & $\begin{array}{l}0.53-2.6 \\
0.46-2.3\end{array}$ & $\begin{array}{l}2.0 \\
1.3\end{array}$ & $\begin{array}{l}0.89-4.4 \\
0.56-3.0\end{array}$ & $\begin{array}{l}1.5 \\
1.8\end{array}$ & $\begin{array}{l}0.59-3.8 \\
0.84-3.8\end{array}$ & $\begin{array}{l}2.0 \\
1.5\end{array}$ & $\begin{array}{l}0.81-4.7 \\
0.65-3.4\end{array}$ \\
\hline 8 Wrist tenderness & 2.8 & & & & & & & & \\
\hline $\begin{array}{l}\text { High/low } \\
\text { Medium/low }\end{array}$ & & $\begin{array}{l}0.93 \\
1.1\end{array}$ & $\begin{array}{l}0.11-8.0 \\
0.13-8.7\end{array}$ & $\begin{array}{l}0.41 \\
1.8\end{array}$ & $\begin{array}{r}0.035-4.8 \\
0.25-14\end{array}$ & $\begin{array}{l}3.7 \\
0.0\end{array}$ & $\begin{array}{c}0.72-19 \\
{ }^{-19}\end{array}$ & $\begin{array}{l}.6 \mathrm{~b} \\
. .6\end{array}$ &.. \\
\hline 9 Frohse's arc local tenderness & 16 & & & & & & & & \\
\hline $\begin{array}{l}\text { High/low } \\
\text { Medium/low }\end{array}$ & & $\begin{array}{l}1.7 \\
2.1\end{array}$ & $\begin{array}{l}0.78-3.9 \\
0.95-4.6\end{array}$ & $\begin{array}{l}1.1 \\
0.90\end{array}$ & $\begin{array}{l}0.54-2.4 \\
0.45-1.8\end{array}$ & $\begin{array}{l}3.1^{\mathrm{a}} \\
2.1\end{array}$ & $\begin{array}{r}1.4-7.2 \\
0.97-4.6\end{array}$ & $\begin{array}{l}2.2^{\mathrm{a}} \\
1.7\end{array}$ & $\begin{array}{r}1.0-4.9 \\
0.75-3.7\end{array}$ \\
\hline 10 Frohse's arc radiating pain & 3.4 & & & & & & & & \\
\hline $\begin{array}{l}\text { High/low } \\
\text { Medium/low }\end{array}$ & & $\begin{array}{l}1.3 \\
3.4\end{array}$ & $\begin{array}{l}0.13-14 \\
0.39-29\end{array}$ & $\begin{array}{l}0.53 \\
0.35\end{array}$ & $\begin{array}{r}0.13-2.2 \\
0.069-1.8\end{array}$ & $\begin{array}{l}2.3 \\
0.95\end{array}$ & $\begin{array}{l}0.33-16 \\
0.16-5.8\end{array}$ & $\begin{array}{l}1.0 \\
1.2\end{array}$ & $\begin{array}{l}0.19-5.3 \\
0.20-7.1\end{array}$ \\
\hline 11 Abduction external rotation test & 13 & & & & & & & & \\
\hline $\begin{array}{l}\text { High/low } \\
\text { Medium/low }\end{array}$ & & $\begin{array}{l}0.90 \\
0.76\end{array}$ & $\begin{array}{l}0.42-1.9 \\
0.36-1.6\end{array}$ & $\begin{array}{l}0.74 \\
0.48\end{array}$ & $\begin{array}{l}0.34-1.6 \\
0.21-1.1\end{array}$ & $\begin{array}{l}1.1 \\
1.4\end{array}$ & $\begin{array}{l}0.43-3.1 \\
0.62-3.0\end{array}$ & $\begin{array}{l}1.3 \\
0.98\end{array}$ & $\begin{array}{l}0.62-2.8 \\
0.45-2.1\end{array}$ \\
\hline 12 Nerve compression tests (5 items) & 20 & & & & & & & & \\
\hline $\begin{array}{l}\text { High//ow } \\
\text { Medium/low }\end{array}$ & & $\begin{array}{l}1.1 \\
1.1\end{array}$ & $\begin{array}{l}0.59-2.0 \\
0.62-2.0\end{array}$ & $\begin{array}{l}0.94 \\
0.59\end{array}$ & $\begin{array}{l}0.51-1.7 \\
0.32-1.1\end{array}$ & $\begin{array}{l}1.8 \\
1.3\end{array}$ & $\begin{array}{l}0.89-3.5 \\
0.68-2.5\end{array}$ & $\begin{array}{l}1.5 \\
1.2\end{array}$ & $\begin{array}{l}0.81-2.8 \\
0.64-2.2\end{array}$ \\
\hline 13 Erector spine tenderness & 17 & & & & & & & & \\
\hline $\begin{array}{l}\text { High/low } \\
\text { Medium/low }\end{array}$ & & $\begin{array}{l}1.3 \\
0.99\end{array}$ & $\begin{array}{l}0.65-2.8 \\
0.47-2.1\end{array}$ & $\begin{array}{l}1.5 \\
1.4\end{array}$ & $\begin{array}{l}0.75-3.0 \\
0.71-2.8\end{array}$ & $\begin{array}{l}2.9^{a} \\
1.5\end{array}$ & $\begin{array}{r}1.2-6.9 \\
0.68-3.3\end{array}$ & $\begin{array}{l}1.7 \\
0.51\end{array}$ & $\begin{array}{l}0.89-3.1 \\
0.21-1.3\end{array}$ \\
\hline 14 Thoracic back tenderness ( 2 items) & 23 & & & & & & & & \\
\hline $\begin{array}{l}\text { High/low } \\
\text { Medium/low }\end{array}$ & & $\begin{array}{l}1.3 \\
0.70\end{array}$ & $\begin{array}{l}0.78-2.3 \\
0.39-1.3\end{array}$ & $\begin{array}{l}1.5 \\
1.8\end{array}$ & $\begin{array}{r}0.83-2.9 \\
1.0-3.3\end{array}$ & $\begin{array}{l}3.7^{\mathrm{a}} \\
2.1\end{array}$ & $\begin{array}{l}1.7-7.8 \\
1.1-4.1\end{array}$ & $\begin{array}{l}1.6 \\
1.2\end{array}$ & $\begin{array}{l}0.87-2.7 \\
0.64-2.2\end{array}$ \\
\hline 15 Lumbar back tenderness ( 3 items) & 26 & & & & & & & & \\
\hline $\begin{array}{l}\text { High/low } \\
\text { Medium/low }\end{array}$ & & $\begin{array}{l}1.8^{a} \\
1.4\end{array}$ & $\begin{array}{r}1.0-3.2 \\
0.77-2.4\end{array}$ & $\begin{array}{l}1.1 \\
0.99\end{array}$ & $\begin{array}{l}0.63-1.8 \\
0.60-1.6\end{array}$ & $\begin{array}{l}1.7 \\
1.2\end{array}$ & $\begin{array}{l}0.95-3.1 \\
0.72-2.1\end{array}$ & $\begin{array}{l}1.6 \\
0.86\end{array}$ & $\begin{array}{l}0.96-2.6 \\
0.47-1.6\end{array}$ \\
\hline 16 Spine lateral bending restriction & 35 & & & & & & & & \\
\hline $\begin{array}{l}\text { High/low } \\
\text { Medium/low }\end{array}$ & & $\begin{array}{l}0.86 \\
0.89\end{array}$ & $\begin{array}{l}0.59-1.3 \\
0.63-1.2\end{array}$ & $\begin{array}{l}0.84 \\
1.3\end{array}$ & $\begin{array}{l}0.55-1.3 \\
0.91-1.7\end{array}$ & $\begin{array}{l}0.71 \\
0.85\end{array}$ & $\begin{array}{l}0.46-1.1 \\
0.62-1.2\end{array}$ & $\begin{array}{l}0.86 \\
0.94\end{array}$ & $\begin{array}{l}0.58-1.2 \\
0.67-1.3\end{array}$ \\
\hline 17 Decreased sensitivity in the legs & 4.0 & & & & & & & & \\
\hline $\begin{array}{l}\text { High/low } \\
\text { Medium/low }\end{array}$ & & $\begin{array}{l}1.0 \\
0.96\end{array}$ & $\begin{array}{l}0.27-3.9 \\
0.21-4.4\end{array}$ & $\begin{array}{l}1.4 \\
0.97\end{array}$ & $\begin{array}{l}0.18-12 \\
0.20-4.7\end{array}$ & $\begin{array}{l}2.0 \\
4.1\end{array}$ & $\begin{array}{l}0.20-19 \\
0.79-21\end{array}$ & $\begin{array}{l}2.8 \\
1.6\end{array}$ & $\begin{array}{l}0.77-10 \\
0.29-9.3\end{array}$ \\
\hline 18 Hip tenderness & 8.0 & & & & & & & & \\
\hline $\begin{array}{l}\text { High/low } \\
\text { Medium/low }\end{array}$ & & $\begin{array}{l}1.9 \\
3.3\end{array}$ & $\begin{array}{c}0.45-7.9 \\
1.0-10\end{array}$ & $\begin{array}{l}0.43 \\
0.73\end{array}$ & $\begin{array}{l}0.14-1.3 \\
0.31-1.7\end{array}$ & $\begin{array}{l}1.2 \\
1.6\end{array}$ & $\begin{array}{l}0.26-5.5 \\
0.59-4.1\end{array}$ & $\begin{array}{l}0.59 \\
0.71\end{array}$ & $\begin{array}{l}0.22-1.6 \\
0.28-1.8\end{array}$ \\
\hline 19 Knee tenderness & 13 & & & & & & & & \\
\hline $\begin{array}{l}\text { High/low } \\
\text { Medium/low }\end{array}$ & & $\begin{array}{l}0.63 \\
0.47\end{array}$ & $\begin{array}{l}0.30-1.3 \\
0.22-0.99\end{array}$ & $\begin{array}{l}1.0 \\
1.4\end{array}$ & $\begin{array}{l}0.49-2.2 \\
0.67-2.8\end{array}$ & $\begin{array}{l}0.91 \\
0.88\end{array}$ & $\begin{array}{l}0.39-2.1 \\
0.44-1.8\end{array}$ & $\begin{array}{l}0.80 \\
0.94\end{array}$ & $\begin{array}{l}0.37-1.7 \\
0.43-2.1\end{array}$ \\
\hline 20 Foot tenderness & 6.0 & & & & & & & & \\
\hline $\begin{array}{l}\text { High/low } \\
\text { Medium/low }\end{array}$ & & $\begin{array}{l}4.6 \\
5.6\end{array}$ & $\begin{array}{r}0.78-28 \\
1.1-28\end{array}$ & $\begin{array}{l}0.49 \\
1.0\end{array}$ & $\begin{array}{r}0.092-2.6 \\
0.35-3.0\end{array}$ & $\begin{array}{l}2.0 \\
0.70\end{array}$ & $\begin{array}{l}0.66-6.1 \\
0.22-2.2\end{array}$ & $\begin{array}{l}11 \\
14\end{array}$ & $\begin{array}{c}0.31-420 \\
1.9-96\end{array}$ \\
\hline 21 Spine muscle tenderness ( 3 items) & 38 & & & & & & & & \\
\hline $\begin{array}{l}\text { High/low } \\
\text { Medium/low }\end{array}$ & & $\begin{array}{l}1.5^{a} \\
1.2\end{array}$ & $\begin{array}{r}1.0-2.2 \\
0.80-1.8\end{array}$ & $\begin{array}{l}1.2 \\
1.3\end{array}$ & $\begin{array}{l}0.82-1.8 \\
0.88-1.8\end{array}$ & $\begin{array}{l}1.5 \\
1.3\end{array}$ & $\begin{array}{l}0.94-2.3 \\
0.91-1.9\end{array}$ & $\begin{array}{l}1.6^{\mathrm{a}} \\
1.3\end{array}$ & $\begin{array}{r}1.1-2.3 \\
0.89-2.0\end{array}$ \\
\hline 22 Joint tenderness (8 items) & 53 & & & & & & & & \\
\hline $\begin{array}{l}\text { High/low } \\
\text { Medium/low }\end{array}$ & & $\begin{array}{l}1.1 \\
0.98\end{array}$ & $\begin{array}{l}0.86-1.5 \\
0.74-1.3\end{array}$ & $\begin{array}{l}0.95 \\
1.1\end{array}$ & $\begin{array}{l}0.71-1.3 \\
0.87-1.5\end{array}$ & $\begin{array}{l}1.4 \\
1.2\end{array}$ & $\begin{array}{l}0.99-1.9 \\
0.92-1.6\end{array}$ & $\begin{array}{l}1.1 \\
1.1\end{array}$ & $\begin{array}{l}0.81-1.4 \\
0.79-1.5\end{array}$ \\
\hline 23 Nerve affections (6 items) & 23 & & & & & & & & \\
\hline $\begin{array}{l}\text { High/low } \\
\text { Medium/low }\end{array}$ & & $\begin{array}{l}1.3 \\
1.1\end{array}$ & $\begin{array}{l}0.71-2.2 \\
0.61-1.9\end{array}$ & $\begin{array}{l}0.94 \\
0.65\end{array}$ & $\begin{array}{l}0.52-1.7 \\
0.36-1.1\end{array}$ & $\begin{array}{l}1.8 \\
1.6\end{array}$ & $\begin{array}{l}0.92-3.6 \\
0.87-2.9\end{array}$ & $\begin{array}{l}1.6 \\
1.2\end{array}$ & $\begin{array}{l}0.94-2.9 \\
0.68-2.3\end{array}$ \\
\hline
\end{tabular}

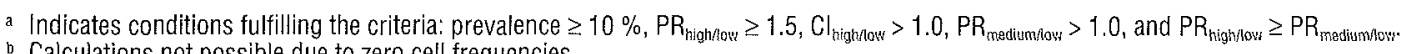

b Calculations not possible due to zero cell frequencies. 


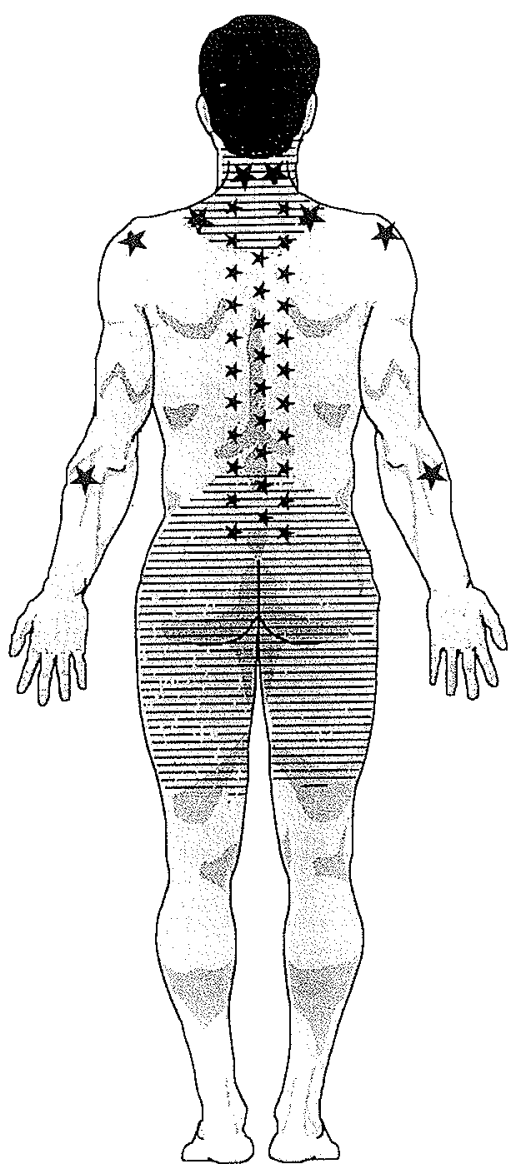

Figure 1. Location of symptoms (striped) and signs (stars) with the most pronounced associations with poor self-rated psychosocial work conditions.

regions, with pronounced associations with symptoms but not with signs. One possible explanation of the latter findings is that symptoms in the lower back are known to refer to the (gluteal and hip) regions shown in the symptom-recording questionnaire.

In congruence with the hypotheses, few associations were noted for the more peripheral regions of the body (ie, the upper or lower extremities). The exception was the pronounced association with local tenderness at Frohse's arc. Palpation of Frohse's arc was done as a test of entrapment of the radial nerve. In addition to the radial nerve, this test affects the wrist and finger extensors and the supinator muscle. Local tenderness was frequently registered and was possibly a sign of muscle (soft-tissue) tenderness. One possibility is that this tenderness was a manifestation of general muscle or soft-tissue tenderness. Symptoms from the arms were associated with poor psychosocial work conditions among production workers but not among office workers in one of the few other studies covering the arm region (14). No correction was however made for physical load in this cited crosssectional study. Psychogenic muscle tension recorded by
EMG has also been reported for flexors in passive forearms during experimentally induced stress (37). Studies regarding the legs or feet have reported diverging associations $(14,38)$.

Few positive findings, except those of the abduction external rotation test, were noted in tests of affections of peripheral nerves. The summary variable of all the tests of nerve affections showed no pronounced association, which is in accordance with the hypotheses. No other studies have been found that report results from tests of nerve affections in connection with psychosocial factors, except one reporting positive associations with sciatica (32).

The findings of associations with tenderness in several locations could tentatively be explained by the existence of "general tenderness" or low-pain thresholds among our subjects. A study of a randomized subgroup of our subjects, on the contrary, however, showed higher pain thresholds among the subjects reporting high mental demands (39).

According to the results of this study, psychosocial work conditions have pronounced associations with symptoms and specific signs in central body regions. If data regarding work-related musculoskeletal disorders (effect measures) are lumped together from different body regions and different signs, true associations could be hidden, as it would have the same effect as nondifferential misclassification of the effect measure with attenuation of the risk estimates as a consequence (40).

Internal consistency and test-retest reliability have been shown to be fairly good regarding the indices "mental demands" and "social support", but not "decision latitude", and therefore can partly explain the consistent lack of association with the latter index. The validity and reliability of the symptom registration instrument was not studied in this investigation, but it is reported to be satisfactory and does not substantially differ between regions of the body $(28,41)$. The reliability of the medical examination was fair, as was mentioned in the methods section, and it did not substantially differ between the examination categories. There were thus no indications that bias was selective regarding body regions or structures. The main results were therefore probably not affected by these sources of error.

The subjects in this study were chosen from different occupations in order to obtain variation in both physical and psychosocial exposure: furniture movers, secretaries, and population samples. Analyses stratifying for subgroup showed the same main pattern as the preceding results, indicating that subject category did not act as a serious confounder. There was still the possibility of an effect modification within some subject category.

As 164 separate comparisons were made between the exposure and effect variables, it can be argued that the 
Table 4. Prevalences of syndromes based on the co-existence of questionnaire-based symptoms and signs in the medical examination of 358 subjects of mixed gender and occupations. Prevalence ratios (PR) with 95\% test-based confidence intervals (95\% Cl) for subjects with high exposure versus subjects with low exposure (high/low) and subjects with medium exposure versus subjects with low exposure (medium/low) to 4 variables of self-rated psychosocial work conditions. PR based on Mantel-Haenszel calculations on data stratified by gender, 2 age groups and low/high physical load at work.

\begin{tabular}{|c|c|c|c|c|c|c|c|c|c|}
\hline \multirow[t]{3}{*}{ Syndrome } & \multirow{3}{*}{$\begin{array}{c}\text { Preva- } \\
\text { lence } \\
(\%)\end{array}$} & \multicolumn{8}{|c|}{ Psychosocial exposure variable } \\
\hline & & \multicolumn{2}{|c|}{$\begin{array}{l}\text { Psychological } \\
\text { demands }\end{array}$} & \multicolumn{2}{|c|}{$\begin{array}{l}\text { Decision } \\
\text { latitude }\end{array}$} & \multicolumn{2}{|c|}{$\begin{array}{l}\text { Social } \\
\text { support }\end{array}$} & \multicolumn{2}{|c|}{$\begin{array}{l}\text { Job } \\
\text { strain }\end{array}$} \\
\hline & & PR & $95 \% \mathrm{Cl}$ & PR & $95 \% \mathrm{Cl}$ & PR & $95 \% \mathrm{Cl}$ & $\mathrm{PR}$ & $95 \% \mathrm{Cl}$ \\
\hline 1 Tension neck & 9.9 & & & & & & & & \\
\hline $\begin{array}{l}\text { High/low } \\
\text { Medium/low }\end{array}$ & & $\begin{array}{l}1.7 \\
0.70\end{array}$ & $\begin{array}{l}0.65-4.6 \\
0.23-2.1\end{array}$ & $\begin{array}{l}1.3 \\
1.5\end{array}$ & $\begin{array}{l}0.50-3.4 \\
0.57-3.9\end{array}$ & $\begin{array}{l}2.7^{\mathrm{a}} \\
1.0\end{array}$ & $\begin{array}{r}1.1-6.7 \\
0.37-3.0\end{array}$ & $\begin{array}{l}2.3 \\
1.7\end{array}$ & $\begin{array}{l}0.83-6.6 \\
0.51-6.0\end{array}$ \\
\hline 2 Tendalgia upp.extrem. & 3.1 & & & & & & & & \\
\hline $\begin{array}{l}\text { High/low } \\
\text { Medium/low }\end{array}$ & & $\begin{array}{l}1.8 \\
2.5\end{array}$ & $\begin{array}{l}0.20-16 \\
0.24-27\end{array}$ & $\begin{array}{l}3.0 \\
1.3\end{array}$ & $\begin{array}{l}0.50-18 \\
0.14-11\end{array}$ & $\begin{array}{l}. . \mathrm{b} \\
. . \mathrm{b}\end{array}$ & $\begin{array}{l}. . \\
. .\end{array}$ & $\begin{array}{l}.60 \\
.6\end{array}$ & $\begin{array}{l}. . \\
. .\end{array}$ \\
\hline 3 Nerve compression syndrome & 7.2 & & & & & & & & \\
\hline $\begin{array}{l}\text { High/low } \\
\text { Medium/low }\end{array}$ & & $\begin{array}{l}1.0 \\
0.99\end{array}$ & $\begin{array}{l}0.33-3.0 \\
0.30-3.3\end{array}$ & $\begin{array}{l}1.0 \\
0.59\end{array}$ & $\begin{array}{l}0.31-3.4 \\
0.18-1.9\end{array}$ & $\begin{array}{l}2.4 \\
1.7\end{array}$ & $\begin{array}{l}0.66-8.8 \\
0.50-6.2\end{array}$ & $\begin{array}{l}1.7 \\
1.2\end{array}$ & $\begin{array}{l}0.55-5.1 \\
0.43-3.7\end{array}$ \\
\hline 4 Lumbalgia & 11 & & & & & & & & \\
\hline $\begin{array}{l}\text { High/low } \\
\text { Medium/low }\end{array}$ & & $\begin{array}{l}2.8 \\
1.3\end{array}$ & $\begin{array}{l}0.88-9.0 \\
0.38-4.3\end{array}$ & $\begin{array}{l}1.7 \\
1.2\end{array}$ & $\begin{array}{l}0.65-4.3 \\
0.41-3.5\end{array}$ & $\begin{array}{l}3.7^{\mathrm{a}} \\
2.2\end{array}$ & $\begin{array}{c}1.0-13 \\
0.68-7.1\end{array}$ & $\begin{array}{l}2.2 \\
1.3\end{array}$ & $\begin{array}{l}0.86-5.7 \\
0.40-4.0\end{array}$ \\
\hline 5 Tendalgia low.extrem. & 8.2 & & & & & & & & \\
\hline $\begin{array}{l}\text { High/low } \\
\text { Medium/low }\end{array}$ & & $\begin{array}{l}0.59 \\
0.87\end{array}$ & $\begin{array}{l}0.16-2.1 \\
0.32-2.4\end{array}$ & $\begin{array}{l}0.65 \\
0.46\end{array}$ & $\begin{array}{l}0.29-1.4 \\
0.20-1.1\end{array}$ & $\begin{array}{l}1.8 \\
0.83\end{array}$ & $\begin{array}{l}0.49-6.8 \\
0.29-2.3\end{array}$ & $\begin{array}{l}0.42 \\
0.92\end{array}$ & $\begin{array}{l}0.13-1.4 \\
0.33-2.6\end{array}$ \\
\hline
\end{tabular}

a Indicates conditions fulfilling the criteria: $P R_{\text {high/low }} \geq 1.5, \mathrm{Cl}_{\text {high/low }}>1.0, P R_{\text {mediumnow }}>1.0$, and $P R_{\text {high/low }} \geq P R_{\text {medium/low. }}$.

b Calculations not possible due to zero cell frequencies.

results of this study can be explained by chance associations alone, an effect of "multiple comparisons". No adjustments of the level of significance were made in this study, as confidence intervals were calculated and as such methods have been criticized (40). However, the criteria for "pronounced association" have a similar conservative effect. The main arguments against chance as an explanation for the results are that they are biologically plausible, have a consistent pattern, and have been tested against a priori hypotheses (40). The included extra medical examination items, which were supposedly less likely to be associated with the exposure variables, showed no such "chance" associations. The findings in our study need verification in future investigations in order to make the interpretation of the results more definite. No cause-effect relations can be concluded from this study due to its cross-sectional design.

\section{Concluding remarks}

Perceived high psychological demands, high job strain, or low social support are statistically associated with both symptoms and signs of the musculoskeletal system. Such associations are the most pronounced with symptoms and signs from central body regions (ie, the neck and low back) in comparison with more peripheral regions such as the arms or legs. The associations are more pronounced with signs of muscular (soft-tissue) tender- ness than with signs of affections of nerves, joints, tendons, or muscular insertions. Studies of associations between psychosocial work conditions and musculoskeletal disorders should separate effect measures of different clinical signs and different body regions in order to avoid attenuation of the risk estimates.

\section{Acknowledgments}

This was a part of the Stockholm MUSIC I study that was financially supported by the Swedish Work Environment Fund, Folksam Insurance Group, and Stockholm County Council. Professor Mats Hagberg, National Institute of Occupational Health, the Director of the Stockholm MUSIC I study, has given valuable advice and comments for this work. Dr Gunnar Németh, Karolinska Hospital, guided the medical examinations and provided valuable comments to this study. Dr Lars Alfredsson, Institute of Environmental Medicine at the Karolinska Institute, also provided valuable comments to this manuscript. Epidemiologist Max Köster, Karolinska Hospital, Department of Occupational Health, was of great help with the data processing.

\section{References}

1. Armstrong TJ, Buckle P, Fine LJ, Hagberg M, Jonsson B, Kilbom $\AA$, et al. A conceptual model for work-related neck 
and upper-limb musculoskeletal disorders. Scand J Work Environ Health 1993;19:73-84.

2. Hagberg M, Silverstein B, Wells R, Smith MJ, Hendrick HW, Carayon $\mathrm{P}$, et al. Work related musculoskeletal disorders (WMSDs): a reference book for prevention; 1st edition. London: Taylor \& Francis, 1995:17-137.

3. Bongers PM, de Winter CR, Kompier MAJ, Hildebrandt VH. Psychosocial factors at work and musculoskeletal disease. Scand J Work Environ Health 1993;19:297-312.

4. Lindström K. Psychosocial criteria for good work organization. Scand J Work Environ Health 1994;20 special issue: $123-33$.

5. Karasek R, Theorell T. Healthy work: stress, productivity and the reconstruction of working life. New York (NY): Basic Books Inc, 1990.

6. Johnson JV, Hall EM. Job strain, workplace social support and cardiovascular disease: a cross-sectional study of a random sample of the Swedish working population. Am J Public Health 1988;78:1336-42.

7. Theorell T, Harms-Ringdahl K, Ahlberg-Hultén G, Westin B. Psychosocial job factors and symptoms from the locomotor system - a multicausal analysis. Scand J Rehabil Med 1991; $23: 165-73$.

8. Ahlberg-Hultén G, Theorell T, Sigala F. Social support, job strain and musculoskeletal pain among female health care personnel. Scand J Work Environ Health 1995;21:435-9.

9. Ursin H, Endresen I, Svebak S. Muscle pain and coping with working life in Norway: a review. Work Stress 1993;7:247_ 58.

10. Veiersted KB. Stereotyped light manual work, individual factors and trapezius myalgia [dissertation]. Oslo: Department Group for Community Medicine, University of Oslo, 1995 : $77 \mathrm{p}$.

11. Elert J, Rantapää-Dahlqvist S, Almay B, Eisemann M. Muscle endurance, muscle tension and personality traits in patients with muscle or joint pain - a pilot study. J Rheumatol 1993; 20:1550-6.

12. Vasseljen O. Work-related shoulder and neck pain with reference to muscle activity, individual factors, and psychosocial exposures [dissertation]. Trondheim (Norway): The Norwegian Institute of Technology, Division of Organization and Work Science, University of Trondheim, 1995:150 p.

13. Reich W. Character analysis, 3rd edition. London: Lowe \& Brydone, 1948.

14. Westgaard RH, Jensen C, Hansen K. Individual and workrelated risk factors associated with symptoms of musculoskeletal complaints. Int Arch Occup Environ Health 1993;64: $405-13$.

15. von Ludwig J. Psychoanalytisch-psychophysiologische untersuchungen bei patienten mit funktionellen cervikalsyndrom Ztg Psychosom Med 1978;24:101-15.

16. Flor H, Turk DC, Birbaumer N. Assessment of stress-related psychophysiological reactions in chronic back pain patients. J Consult Clin Psychol 1985;53:354 64 .

17. Flor H, Birbaumer N, Schugens MM, Lutzenberger W. Symptom-specific psychophysiological responses in chronic pain patients. Psychophysiol 1992;29:452-60.

18. Shagass C, Malmo RB. Psychodynamic themes and localized muscular tension during psychotherapy. Psychosom Med 1954;16:295-314.

19. Shipman WG, Oken D, Balshan Goldstein I, Grinker RR, Heath HA. Study in psychophysiology of muscle tension. II personality factors. Arch Gen Psychiatry 1964;11:330—45.

20. Weber A, Fussler C, O'Hanlon JF, Gierer R, Grandjean E.
Psychophysiological effects of repetitive tasks. Ergonomics 1980;23:1033-46.

21. Westgard RH, Jensen C, Nilsen K. Muscle coordination and choice-reaction time test as indicators of occupational muscle load and shoulder-neck complaints. Eur J Appl Physiol 1993; $67: 106-14$

22. Lundberg U, Kadefors R, Melin B, Palmerud G, Hassmén P Engström $M$, et al. Psychophysiological stress and EMG activity of the trapezius muscle. Int J Behav Med 1994:4 354-70.

23. Ekberg K, Eklund J, Tuvesson M-A, Odenrick P, Ericson M, Örtengren R. Psychic stress and muscle activity during data entry at visual display units. Work Stress 1995;9:475-90.

24. Wærsted M, Bjørklund RA, Westgaard RH. The effect of motivation on shoulder-muscle tension in attention-demanding tasks. Ergonomics 1994;37:363 - 76 .

25. Hagberg M, Goldie I, Ahlbom A, Ekholm J, Gamberale F, Gullberg R, et al. Plan and operation of the Stockholm-MU SIC I study, prevention of musculoskeletal disorders. In: $23 \mathrm{rd}$ international congress on occupational health [book of abstracts]. Montréal (Canada): International Commission of Occupational Health, 1990:645.

26. Theorell T, Ahlberg-Hultén G, Sigala F, Perski A, Söderholm M, Kallner A, et al. A psychosocial and biomedical comparison between men in six contrasting service occupations. Work Stress 1990;4:51-63.

27. Theorell T, Perski A, Åkerstedt T, Sigala F, Ahlberg-Hultén $G$, Svensson J, et al. Changes in job strain in relation to changes in physiological state. Scand J Work Environ Health 1988;14:189-96.

28. Kuorinka I, Jonsson B, Kilbom $\AA$, Vinterberg H, BieringSorensen F, Andersson G, et al. Standardised Nordic questionnaires for the analysis of musculoskeletal symptoms. Appl Ergon 1987;18:233-7.

29. Mantel N, Haenszel W. Statistical aspects of analysis of data from retrospective studies of disease. JNCI 1959;22:719 48 .

30. Miettinen OS. Estimability and estimation in case-referent studies. Am J Epidemiol 1976;103:226 - 35.

31. Linton SJ. Risk factors for neck and back pain in a working population in Sweden. Work Stress 1990;4:41—9.

32. Mäkelä M. Common musculoskeletal syndromes: prevalence, risk indicators and disability in Finland [dissertation]. Helsinki: Department of Public Health Science, University of Helsinki, 1993:162 p.

33. Houtman ILD, Bongers PM, Smulders PGW, Kompier MAJ. Psychosocial stressors at work and musculoskeletal problems. Scand J Work Environ Health 1994;20:139-45.

34. Johansson JÅ. Psychosocial factors at work and their relation to musculoskeletal symptoms [dissertation]. Göteborg (Sweden): Department of Psychology, University of Göteborg, 1994:118 p.

35. Veiersted KB, Westgaard RH. Subjectively assessed occupational and individual parameters as risk factors for trapezius myalgia. Int J Ind Ergon 1994;13:235-45.

36. Leino PI, Hänninen V. Psychosocial factors at work in relation to back and limb disorders. Scand J Work Environ Health 1995;21:134-42.

37. Svebak S, Braathen ET, Sejersted OM, Bowim B, Fauske S, Laberg J. Electromyographic activation and proportion of fast versus slow twitch muscle fibers: a genetic disposition for psychogenic muscle tension? Int J Psychophysiol 1993;15: $43-9$.

38. Bergenudd $H$, Nilsson $B$, Lindgärde $F$. Knee pain in middle age and its relationship to occupational work load and psycho- 
social factors. Clin Orthop 1989:210-15.

39. Theorell T, Nordemar R, Michélsen H, Stockholm MUSIC I Study Group. Pain thresholds during standardized psychological stress in relation to perceived psychosocial work situation. J Psychosom Res 1993;37:299--305.

40. Rothman KJ. Modern epidemiology. 1st edition. Boston (MA): Little, Brown and Company, 1986.
41. Ohlsson K, Attewell RG, Johnsson B, Ahlm A, Skerfving S. An assessment of neck and upper extremity disorders by questionnaire and clinical examination. Ergonomics 1994;37: $891-7$.

Received for publication: 1 February 1996 